\title{
OCA-MAC: Opportunistic Channel Aggregation for Wireless Ad Hoc Networks
}

\author{
Larissa E. de Oliveira* Luiz A. DaSilva ${ }^{\dagger}$ Marcelo M. Carvalho* \\ Department of Electrical Engineering* \\ University of Brasília, Brazil \\ Department of Electrical and Computer Engineering ${ }^{\dagger}$ \\ Virginia Polytechnic Institute and State University, USA
}

\begin{abstract}
This paper introduces the Opportunistic Channel Aggregation MAC protocol (OCA-MAC) for wireless ad hoc networks. OCA-MAC allows opportunistic MAC-level channel aggregation per frame transmission. To accomplish that, OCAMAC is built on top of the IEEE 802.11 DCF MAC with a secondary radio interface for opportunistic access over an alternative channel. OCA-MAC avoids the use of extra control channel for coordination among nodes (or control frame exchanges over the secondary channel) by using the simple idea that whoever acquires the floor over the primary channel also gains immediate (and implicit) right to transmit a frame over the secondary channel (if available at both sides of the link at the time of floor acquisition). Thus, all control information for distributed channel aggregation is carried out over the primary channel, allowing the use of the secondary channel for data frame transmissions only. The gains in performance of OCA-MAC with respect to IEEE 802.11 DCF MAC is evaluated by simulations with topologies not fully connected under saturated traffic. The investigated scenarios indicate that geographical sparsity and spatial reuse do not necessarily lead to performance improvements of IEEE 802.11 networks and, thus, opportunistic channel aggregation proves to be a strong ally to boost throughput and fairness performance in such scenarios.
\end{abstract}

\section{INTRODUCTION}

The advent of multi-interface wireless devices, coupled with current efforts to open up frequency bands to accomodate the volume of mobile data traffic, have ignited a true revolution in the design of future wireless networks. In particular, the success of the IEEE 802.11 standard has pushed the limits of current ISM bands to efficiently absorb a multitude of WLANs and other technologies. In particular, the availability of multiple channels has inspired the design of new multi-channel MAC protocols as a means to achieve higher throughput by contention alleviation and self-interference avoidance.

In the realm of wireless ad hoc networks, the design of multi-channel MAC protocols has recently followed the path of cognitive MAC protocols, where the ad hoc network is tipically regarded as a "secondary" network that seeks to transmit opportunistically over vacant channels assigned to other "primary" network. In such a scenario, a major problem consists of devising smart solutions for channel search and negotiaton with intended receiver(s). As a result, the understanding of the primary user's channel occupation behavior is also a key information that needs to be taken into account in the design of MAC protocols. However, since the scenarios originally envisioned for ad hoc networks as "secondary users" have not become a reality yet (or it is still unclear whether they will be allowed in future spectrum regulations), a more urgent need under today's shortage of ISM spectrum is to leverage the availability of multiple channels on current ISM bands so that the devices can boost their throughput performance by opportunistic channel aggregation. Curiously, most of previous work on multi-channel MAC protocols for ad hoc networks have only targeted the distributed assignment of non-overlapping channels as a means to promote concurrent transmissions and alleviate self-interference [1], [2], [3], [4], [5], [6], [7]. But, few attempts have been made to expand a node's throughput by channel aggregation. The concept of channel aggregation is not new, as it has been recently adopted in wireless standards such as LTE. For that, many techniques have been devised at the physical (PHY) layer, specially through the use of OFDM modulation. However, achieving the granularity of channel aggregation allowed by OFDM requires sofisticated channel monitoring and aggregation techniques. In current scenarios where multiple ISM channels are available, one may be interested in performing channel aggregation at the $M A C$ level, as a way to improve throughput without requiring much complexity at the PHY layer.

With this goal in mind, we introduce OCA-MAC, a MAC protocol for ad hoc networks that performs (MAC-level) opportunistic channel aggregation per frame transmission. OCAMAC is built on top of the IEEE 802.11 DCF MAC and relies on the availability of a secondary radio interface for opportunistic access over an alternative channel. OCA-MAC neither uses extra control channel(s) nor depends on control frame exchanges over the secondary channel for coordination among nodes. Instead, it is based on the simple idea that whoever acquires the floor over the primary channel, also gains immediate (and implicit) right to transmit a data frame over the secondary channel (if available at both sides of the link at the time of floor acquisition). Thus, all control information for distributed channel aggregation is carried out over the primary channel, which allows the use of the secondary channel for data frame transmissions only. We evaluate the gains in performance of OCA-MAC over standard single-channel IEEE 802.11 networks with topologies not fully connected under saturated traffic. 


\section{The OCA-MAC PRotocol}

The Opportunistic Channel Aggregation MAC protocol (OCA-MAC) is built on top of the IEEE 802.11 DCF MAC in order to extend its basic functionalities to allow opportunistic MAC-level channel aggregation. By MAC-level channel aggregation one should understand as the use of a secondary channel for concurrent DATA frame transmissions with the primary channel. For channel aggregation, it is assumed that each node is equipped with two radio transceivers: one to be tuned to a given ISM channel, and another that can be tuned to a different, non-overlapping ISM channel. In principle, OCAMAC could also operate in other frequency bands, as long as the conditions to be described next are satisfied for correct protocol operation. We focus on channels of a given ISM band, since they provide similar propagation characteristics.

All nodes under OCA-MAC compete for channel access over the primary ISM channel according to the traditional four-way handshake mechanism and binary exponential backoff (BEB) algorithm of the IEEE 802.11 DCF MAC. Hence, with the sending of an RTS, a node simultaneously attempts to reserve the primary ISM channel for transmission of a DATA frame $i$, and to establish a secondary channel to be used for opportunistic transmission of a DATA frame $i+1$. If the receiver accepts transmission over the proposed channel (informed in the RTS), and the following CTS is received successfully by the sender, two DATA frames are transmitted simultaneously over both interfaces. It is assumed that channel availability at both interfaces is evaluated according to standard energy detection strategies (i.e., no advanced signal detection algorithms are assumed).

One key element for operation of OCA-MAC is a control module that is responsible for appropriate handling of DATA frames to be transmitted (and received) at both interfaces. This module acts as an interface between the network and MAC layers. It contains a transmit buffer to enqueue every datagram received from the network layer. This buffer acts as a prebuffer to the buffers located at the MAC layer. Also, the control module is responsible for any re-ordering of frames before delivery to the destination's network layer. To convey control information about opportunistic transmissions, additional fields are added to the RTS, CTS, and ACK frames of the IEEE 802.11 DCF. The RTS contains the request available channel (RAC) field, in which the sender informs which opportunistic channel(s) it has found available for immediate exchange of data. Hence, we assume that the RAC field contains $k$ bits that can be used in any way to express availability of a single or, possibly, many channels. The specific mapping of the $2^{k}$ bit sequences into frequency channels must be tailored to the operational frequency range for which the control module was designed for. By convention, the RAC field is filled with the all-zero bit sequence if no channel is available.

Upon reception of the RTS, the receiver reads the RAC field and checks for availability of the proposed opportunistic channel(s). This step is necessary because a channel that appears available to the sender may not be available around the receiver. Also, during RTS transmission, another node under OCA-MAC may have occupied the proposed channel(s) or, alternatively, the sender may have made an incorrect decision on channel availability due to a false positive (e.g., failed signal detection due to channel propagation effects). By acting this way, the receiver also helps to minimize the possibility of interference with any ongoing activity in the proposed channel(s). After checking for availability of the proposed channel(s), the receiver generates a CTS frame with an additional confirm available channel (CAC) field containing its decision regarding the proposed channel(s). In this work, we do not address multiple frame transmissions over multiple channels. Therefore, the receiver only indicates the selected channel (if any) for frame reception. In other words, the CAC field is used to either accept transmission over one of the proposed channel(s) - in which case the CAC field is filled in with information regarding the specific channel—or it denies transmission over the proposed channel by sending the all-zero bit sequence corresponding to channel unavailability.

Once the transmitter receives the CTS, it reads the CAC field. If the CAC field contains the all-zero bit sequence, no transmission occurs over an opportunistic channel. Otherwise, it signals the control module to dispatch a copy of the next datagram in the pre-buffer for immediate transmission through the opportunistic channel. After an appropriate SIFS time interval, two DATA frames are transmitted simultaneously over both interfaces. Here, it is important to mention that, for correct operation, the transmission time over the opportunistic channel must be less than or equal to the transmission time of the DATA frame transmitted over the primary channel. This is because all control information is carried out over the primary channel, including the transmission time needed for virtual carrier sensing through NAV updates. Therefore, a necessary condition for correct operation of OCA-MAC is that the size of the frame to be transmitted opportunistically must be equal to (or smaller than) the size of the frame to be transmitted over the primary channel (assuming same modulation schemes at both interfaces). Future versions of OCA-MAC may allow adaptive modulation schemes to speed up time transmission over the opportunistic channel (with appropriate control functionality to negotiate such options).

If both DATA frames are received successfully, an ACK is sent back to the sender with an additional opportunistic acknowledgment (OPACK) field set to ' 1 ', indicating successful reception on the opportunistic channel. If, on the other hand, any of the DATA frames are not received successfully, two situations may arise: if a successful transmission happens only over the primary channel, then the OPACK field is set to ' 0 ', indicating a failure of the opportunistic transmission. In this case, the next datagram to be transmitted over the primary channel is the one that just failed to be transmitted opportunistically over the secondary channel (hence, the need to keep a copy of the datagram at the pre-buffer). If the failure happens over the primary channel, different approaches may be adopted, depending on whether reception of out-of-order frames is allowed at the receiver side. The simplest approach 
is to accept only frames in order, i.e., to discard any successful transmission over the opportunistic channel if the transmission over the primary channel fails (since this is a subsequent datagram to the one incorrectly received over the primary channel). In this case, less storage and control are required at the receiver side. Another option is to accept out-of-order frames at the receiver side. In this case, appropriate handling of correctly received frames at the secondary channel would be needed, demanding more resources and complex control. In this work, the former approach is adopted due to its simplicity.

Since reception of out-of-order frames is not allowed, both DATA frames are retransmitted if a DATA frame transmission fails over the primary channel. In this case, after execution of the back-off algorithm at the primary interface only, the sending of a new RTS follows the steps previously described. Finally, if the transmitter's RAC field already contains the allzero bit sequence (i.e., the transmitter could not find any opportunistic channel), the receiver simply replies with the same reserved bit sequence. In this case, no channel aggregation occurs. Finally, in order to avoid unnecessary ARP requests on secondary channels, another field is added to the CTS frame: the confirm MAC address (CMA), by which the receiver can convey the MAC address of its secondary interface.

As a final note, it is worth to mention that OCA-MAC seeks in-order delivery of frames, and may be potentially used in aforementioned cognitive scenarios. Note that splitting flows between two independent 802.11 interfaces does not guarantee in-order delivery of frames at the receiver (a basic requirement of any ARQ strategy), and it would require complex flow reassembly at the receiver (such a situation could be worsened in cognitive scenarios, where the presence of the primary user for long periods of time could compromise the reassembly process).

\section{Performance Evaluation}

In this section, we investigate the benefits of MAC-level channel aggreation through OCA-MAC by understanding the performance gains it provides with respect to standard singlechannel IEEE 802.11 b. For this purpose, two non-overlapping ISM channels are defined according to IEEE 802.11 specifications. For performance evaluation, we consider MAC-level aggregate throughput, i.e., the total number of bits successfully transferred across all links in the network, during a certain period of time. This figure of merit gives an idea of the spatial reuse in the network (simultaneous transmissions). By MAC-level throughput we mean the data throughput (in b/s) achieved by the MAC protocol over each link, i.e., the endto-end throughput over a path is not treated in this work. The focus is on static scenarios under saturated traffic in order to investigate the impact of node contention on throughput. Hence, every node always has a data frame ready to be sent on its pre-buffer, while, at the same time, it is the target receiver of some other node. Nine topologies are used with 100 nodes placed on a flat terrain of $1600 \times 1600 \mathrm{~m}$. Transmitter/receiver pairs are located within a maximum transmission range of $150 \mathrm{~m}$ under the two-ray path loss propagation model. Each simulation run corresponds to 60 seconds of CBR traffic using UDP. Ten simulation runs (different seeds) were executed for each topology. Results are reported with a $95 \%$ confidence interval. Table I gives the PHY and MAC parameters used in simulations with the Network Simulator 3 [8]. Both channels use the same parameters.

TABLE I

PHY AND MAC PARAMETERS USED IN SIMULATIONS.

\begin{tabular}{|c|c||c|c|}
\hline \multicolumn{2}{|c||}{ MAC } & \multicolumn{2}{c|}{ PHY } \\
\hline Payload & 1000 bytes & Energy Threshold & $-76.36232 \mathrm{dBm}$ \\
\hline SIFS $_{\text {std }}$ & $16 \mu \mathrm{s}$ & CCA Threshold 1 & $-76.36232 \mathrm{dBm}$ \\
\hline SIFS $_{\text {cog }}$ & $26 \mu \mathrm{s}$ & CCA Threshold 2 & $-81.35987 \mathrm{dBm}$ \\
\hline DIFS & $60 \mu \mathrm{s}$ & Transmission Power & $10 \mathrm{dBm}$ \\
\hline MAC Header & 24 bytes & Noise Figure & $7 \mathrm{~dB}$ \\
\hline RTS & 44 bytes & Antenna Height & $1.04 \mathrm{~m}$ \\
\hline CTS & 46 bytes & Modulation & DBPSK \\
\hline ACK & 38 bytes & Rate & $1 \mathrm{Mbps}$ \\
\hline
\end{tabular}

For evaluation under different contention levels, the used topologies can be loosely divided into three groups: lowcontention topologies, where nodes are sparsely distributed over the terrain, medium-contention topologies, which have a higher degree of connection among neighboring nodes, and high-contention topologies. In addition, because OCA-MAC relies on the clear channel assessment (CCA) mechanism, we also evaluate the impact of the carrier sensing range on network throughput. For that, the same set of topologies are studied under two carrier sensing ranges. In the first case, the CCA threshold is equal to the energy detection threshold, which is a parameter that controls the effective transmission range. In this first case, the carrier sensing range is also set to $150 \mathrm{~m}$. In the second case, the carrier sensing range is set to $200 \mathrm{~m}$, which reflects the most typical scenario (sensing range higher than the transmission range).

Figure 1(a) depicts the aggregate throughput for each topology group when carrier sensing range is $150 \mathrm{~m}$. This case targets higher spatial reuse in spite of (potential) lower signal-to-interference-plus-noise (SINR) at a receiver due to higher interference from nearby transmitters. Under high contention, the gain in aggregate throughput with respect to IEEE 802.11 is about $22.5 \%$, while the gain for mediumcontention topologies is about $40.5 \%$, and $72 \%$ for the lowcontention topologies. It is interesting to observe that, as contention decreases (i.e., sparsity increases), the aggregate throughput of IEEE 802.11 does not increase significantly. This is because every node in our scenarios act as both transmitter and receiver and traffic is saturated, which keeps channel contention high even under sparse topologies (fewer neighbors, but competition is still high with target receiver, since it is also a transmitter). Therefore, even though the contention induced by surrounding neighbors decreases as the topology becomes more sparse, the maximum achievable throughput does not improve much. On the other hand, with the added opportunistic channel aggregation, a decrease in the number of surrounding interferers leads to an increase in the achievable throughput due to an improvement on the SINR (interferers are located further away). 


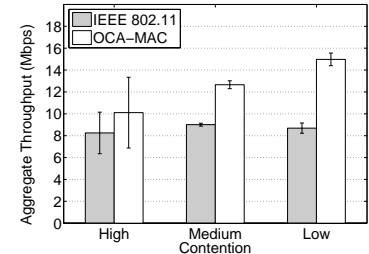

(a)

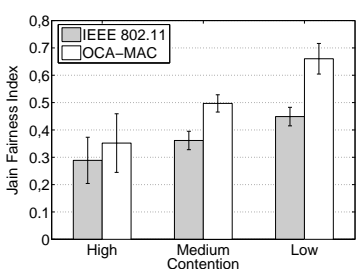

(c)

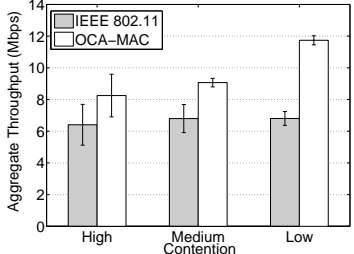

(b)

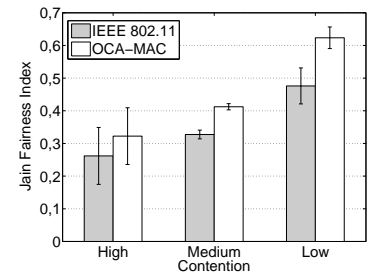

(d)
Fig. 1. (a) Aggregate throughput when carrier sensing range is equal to transmission range. (b) Aggregate throughput when carrier sensing range is $200 \mathrm{~m}$. (c) Fairness index for a carrier sensing range of $150 \mathrm{~m}$. (d) Fairness index for when the carrier sensing range is $200 \mathrm{~m}$.

Figure 1(b) contains the aggregate throughput when carrier sensing range is $200 \mathrm{~m}$. This case corresponds to less spatial reuse, but with high SINR values at receivers. Therefore, the aggregate throughput is lower than the case when the carrier sensing range is $150 \mathrm{~m}$. But, if we look at the performance gains, the scenarios under high contention present an average aggregate throughput gain of $29 \%$, while the average throughput gain under medium-contention topologies is about $33.4 \%$. For low-contention topologies, the average gain in performance is about $72.5 \%$. In other words, OCAMAC mostly benefits from a more conservative clear channel assessment mechanism as far as throughput performance gains with respect to IEEE 802.11 are concerned. When contention is high, and a node acquires the channel, the transmissions over the aggregate channel will have a higher likelihood of being successful due to less interference from distant nodes. The same happens with low-contention topologies, where the more conservative approach guarantees even better conditions over the aggregate channel.

We also investigate the gains in fairness achieved by OCAMAC, since the IEEE 802.11 has serious issues regarding fairness due to its binary exponential backoff algorithm. It is expected that, with OCA-MAC, the nodes who have less chance to acquire the channel will be able to expedite a subsequent frame once the channel is finally acquired, improving overall network fairness. Fairness is computed using Jain's fairness index over each node's average throughput. Figure 1(c) shows the fairness index when carrier sensing range is $150 \mathrm{~m}$. Under high contention, the gains of OCA-MAC over IEEE 802.11 are about $22 \%$, while it increases by $37.5 \%$ under medium contention, and $47 \%$ under low contention. Notice that the fairness of IEEE 802.11 improves as topologies become more sparse. Therefore, although aggregate throughput does not change much, the nodes' individual throughputs become less unequal. Nonetheless, for IEEE 802.11, fairness is still rel- atively poor, and it improves by only $50 \%$ when changing from high- to low-contention scenarios. On the other hand, OCA-MAC presents an improvemente in fairness of $86 \%$ as topologies change from low to higher sparsity. Figure 1(d) displays Jain's fairness index in the case of a 200-m carrier sensing range. With a higher sensing range, nodes will back off more frequently, which leads to fairness degradation by favoring those nodes with stronger links to acquire the channel more frequently. In spite of the higher back-off activity at nodes, OCA-MAC still provides considerable fairness gains over IEEE 802.11.

\section{CONCLUSIONS}

This paper introduced the Opportunistic Channel Aggregation MAC protocol (OCA-MAC) for wireless ad hoc networks. OCA-MAC allows opportunistic MAC-level channel aggregation per frame transmission, and it is built on top of the IEEE 802.11 DCF MAC with the use of a secondary interface for opportunistic access over an alternative channel. No extra channel is needed for coordination among nodes, since it is based on the simple idea that whoever acquires the floor over the primary channel also gains immediate (and implicit) right to transmit a frame over the secondary channel. Simulations have shown that OCA-MAC provides significant gains over IEEE 802.11 with respect to aggregate throughput and fairness, in spite of using only a single, fixed secondary channel for all nodes. Even under sparse topologies, the gains in throughput of IEEE 802.11 are very limited if nodes are saturated. But, with channel aggregation, the increase in sparsity causes less interference from distant nodes, which allows a higher successful frame delivery rate over the secondary channel. This result indicates that spatial reuse and geographical sparsity do not necessarily lead to performance improvements of IEEE 802.11 networks, and opportunistic channel aggregation proves to be a strong ally to boost throughput and fairness performance in such scenarios.

\section{REFERENCES}

[1] Xiaowei Ni, Chunxiu Xu, Muqing Wu, and Jingrong Wen, "DNCA: An efficient channel assignment for multi-interface multi-channel ad hoc wireless networks," in Proc. IEEE Int. Conference on Network Infrastructure and Digital Content (IC-NIDC), 2012, pp. 39-43.

[2] M Maiya and B Hamdaoui, "iMAC: improved medium access control for multi-channel multi-hop wireless networks," Wireless Communications and Mobile Computing, 2011.

[3] Jaeseon Hwang, Taewoon Kim, Jungmin So, and Hyuk Lim, "A receivercentric multi-channel MAC protocol for wireless networks," Computer Communications, vol. 36, no. 4, pp. 431 - 444, 2013.

[4] Xiaochen Lai, Quanli Liu, Wei Wang, Likun Li, Simin Lu, and Ying Zhao, "Dynamic game with perfect and complete information based dynamic channel assignment," Applied Intelligence, pp. 1-13, December 2012.

[5] Yifan Zhang, Qun Li, Gexin Yu, and Baosheng Wang, "ETCH: Efficient channel hopping for communication rendezvous in dynamic spectrum access networks," in Proc. IEEE INFOCOM, 2011, pp. 2471-2479.

[6] Taewoon Kim, Jaeseon Hwang, and Hyuk Lim, "Distributed semisynchronous channel coordination for multi-channel wireless networks," Ad Hoc Networks, vol. 11, no. 1, pp. 368 - 382, 2013.

[7] Shih-Lin Wu, Chih-Yu Lin, Yu-Chee Tseng, and Jang-Ping Sheu, "A new multi-channel MAC protocol with on-demand channel assignment for multi-hop mobile ad hoc network," The Computer Journal, vol. 45, no. 1, pp. 101-110, 2002.

[8] "The ns-3 Network Simulator," http://www.nsnam.org. 